\title{
Estudio del perfil antropométrico del jugador juvenil de balonmano en la Región de Murcia Study Profile Anthropometric Youth Handball Player in Murcia
}

\author{
*Helena Vila Suárez, **J. Arturo Abraldes Valeiras y *Nuria Rodríguez Suárez \\ *Universidad católica San Antonio (España), **Universidad de Murcia (España)
}

Resumen: Determinar la composición corporal y las diferentes características físicas y condicionales fue el primer objetivo planteado en este estudio, donde se valoraron a 45 jugadores de balonmano masculino de la Región de Murcia, correspondiente a la categoría juvenil. Los clubes analizados han sido el Murcia, Alhama, Jumillay Águilas. El segundo objetivo de este trabajo consiste en establecer diferencias entre los equipos valorados y las líneas de juego de carácter ofensivo. El proceso de recogida de datos antropométricos se llevó a cabo siguiendo el protocolo estipulado por el ISAK. El análisis estadístico realizado fue en primer lugar descriptivo y, posteriormente, un análisis inferencial para establecer las diferencias estadísticas entre los distintos equipos y sus líneas de juego, en todas las variables de estudio. La valoración condicional se centró básicamente en la determinación del somatotipo, en la evaluación de la velocidad de lanzamiento y en la fuerza isométrica de tren superior. Los equipos de la parte superior de la tabla presentan valores superiores en las variables antropométricas pero sin ser estadísticamente significativas estas diferencias. Se constata la importancia de la mesomorfia como característica predominante en el somatotipo del jugador de balonmano. Las mayores velocidades se alcanzaron en los lanzamientos en los que hay desplazamiento previo.

Palabra clave: Balonmano, valoración condicional, cineantropometría.

\begin{abstract}
The aim of this study was two fold, first to determine the body composition, physical and conditional characteristics of male handball players, who were playing in juvenile level in Murcia. A Second objective was to establish differences among the teams evaluated and offensive playing lines. The teams analyzed were playing in Murcia, Alhama, Jumilla and Águilas. 45 juvenile male handball players participated in this study. Anthropometric data were collected following ISAK protocols. A descriptive statistical analysis was made in order to obtains means and standard deviation of the simple. An ANOVA analysis was performed in order to establish differences among teams and its playing lines. The somatotype, throwing velocity and grip were analyzed in all teams The best teams show higher values in anthropometric variables than the worts, but these differences does not reach statistical significance. Mesomorphy is a very important characteristic in handball player. Higher throwing velocities were reach in throwings with previous displacements.
\end{abstract}

Key words: Handball, physical evaluation, anthropometry.

\section{Introducción}

El interés, cada vez mayor, por la mejora de los deportes ha llevado a la realización de numerosos trabajos para la evolución del juego, por ello, se analizan y se estudian factores que influyen en el entrenamiento y se crean unas bases que determinen el potencial del jugador para llegar al máximo rendimiento en su actividad específica. El deporte actual necesita un desarrollo científico paralelo, que proporcione una base sólida de análisis, control y valoración del proceso de enseñanza-aprendizaje y, más tarde, del entrenamiento (Zatsiorski, 1989; Verjoshanski, 1990; Martín Acero y Vittori, 1997; García Manso, 1997, Chirosa, 1997).

La inexistencia de un marco teórico capaz de describir y explicar las relaciones de interacción y jerarquía entre los diferentes componentes del rendimiento deportivo, hace necesario investigar todos y cada uno de los aspectos implícitos en el largo camino de la vida deportiva de un jugador, con la finalidad de dotar al profesional de la educación física y del deporte de mayores argumentos para que pueda intervenir con mayor conocimiento de causa-efecto y para poder alcanzar el alto rendimiento deportivo.

Según Seirul-lo (1993) se puede considerar al jugador como una estructura hipercompleja, destacando la presencia de tres estructuras básicas que conforman al sujeto deportista: la estructura condicional, la coordinativa y la cognitiva. La investigación que se presenta a continuación pretende contribuir al estudio científico de un deporte de equipo como el balonmano, centrándose en el análisis de la estructura condicional de deportistas de categoría nacional.

\footnotetext{
Fecha recepción: 20-05-09 - Fecha envío revisores: 20-05-09 - Fecha de aceptación: 18-06-09 Correspondencia: Helena Vila Suárez

Campus de los Jerónimos, s/n

30107 Guadalupe (Murcia)

E-mail: abraldes@um.es
}

La valoración de la estructura condicional (o valoración funcional) consiste en la evaluación objetiva de las capacidades físicas que posee un sujeto para realizar una tarea deportiva (Rodríguez, 1999). Siguiendo a Rodríguez (1999), esta valoración nos permite, entre otros aspectos, obtener información sobre:

- El perfil o modelo de la respuesta funcional que caracteriza a una actividad física o deportiva; es decir, nos da información sobre la participación de las distintas capacidades físicas y vías energéticas en esa tarea deportiva.

- Las diferencias en la respuesta fisiológica de los diferentes individuos, condicionada por variables biológicas como la edad, sexo, peso, etc..

- El establecimiento de elementos objetivos de selección de individuos.

En balonmano, deporte colectivo y de contacto, en elque se realizan esfuerzos de máxima intensidad y corta duración, en los que los jugadores tienen que correr, saltar y lanzar el balón con momentos de reposo o baja intensidad, donde los jugadores realizan acciones contra sus adversarios como bloqueos, golpeos, contactos y empujes (Jacobs, Westlin, Rasmusson y Houghton, 1982).

Los principales tipos de metabolismo que se dan en este deporte son el aeróbico, el anaeróbico aláctico, la fuerza máxima y la potencia muscular, destacando también la velocidad de lanzamiento. (Van Muijen, Joris, Kempery Van Ingen, 1991). Confirmando(Gorostiaga, Granados, Ibáñez, González-Badillo e Izquierdo, 2005) que la capacidad para producir gran cantidad de fuerza y de potencia muscular es lo que define a los jugadores deélite, predominando el metabolismo anaeróbico aláctico.

Estudios recientes (Gorostiaga, et al.) han demostrado las principales diferencias de los deportistas que están en la élite mundial de este deporte con los que compiten en ligas de menor nivel deportivo, destacando la fuerza máxima y potencia de las extremidades superiores e inferiores. Uno de los factores básicos determinantes de la velocidad de lanzamiento es la fuerza y potencia muscular tanto de miembro superior como de miembro inferior, como ya se comentó anteriormente. Para 
incrementar la potencia cuando se posee una técnica estable, es necesario poseer altos niveles tanto de fuerza aplicada como de velocidad en la contracción muscular(Toumi, Best, Martin, Poumarat, 2004; Gorostiaga, Izquierdo, Iturralde, Ruesta y Ibáñez, 1999; Van Muijen, Joris, Kemper y Ingen, 1991; Gorostiaga, y col. 2005; Vicente-Rodríguez, Dorado, Pérez-Gómez, González-Henriquez y Calbet, 2004).

Se han encontrado valores superiores en jugadores de elite $(8 \%)$ respecto a otros de menor categoría (Gorostiaga y col. 2005), y otros estudios realizados en los años 80 y 90 que denotan una mejora históricaen la velocidad de lanzamiento del balón en los jugadores de balonmano de elite (leco, Smith, Craib y Mitchell, 1992; Loftin, Anderson, Lytton, Pittman, y Warren, 1996), hay que reseñar que los métodos utilizados en las diversas pruebas han variado, pasando por células fotoeléctricas llegando hasta el radar, variando referencias y distancias, con lo cual los resultados han de ser tomarlos con cautela.

Aunque existen estudios que ya han demostrado que la fuerza muscular es un factor importante que influye sobre la velocidad de lanzamiento en este deporte en jugadores de elite estos estudios los han mostrado a través de mediciones isocineticas e isométricas y puesto que este tipo de contracción muscular no es en absoluto especifica para un jugador de balonmano, se deberán validar tests dinámicos que si sean más específicos en este tipo de jugadores, y se acerquen más a la especificidad del balonmano (Srhoj, Marinovic, y Rogulj, 2002; Rogulj, Srhoj, Nazor, Srhoj, Cavala., 2005).

Lo que se pretende es reflejar cuales son los métodos y las pruebas físicas que se deben de realizar para comprobar donde está la diferencia entre los jugadores de élite y los demás practicantes. Los valores de cineantropometría, la fuerza máxima y la potencia muscular son parámetros valorados en diferentes trabajos para demostrar donde están las diferencias físicas entre las diferentes poblaciones (Gorostiaga et. $\mathrm{Al}, 2005)$

La fuerza muscular es determinante para la velocidad de lanzamiento (leco, Smith, Craib, Deban Snow y Mitchel, 1992), ya que encuentran relaciones significativas entre la velocidad de lanzamiento y la fuerza ejercida, además de la fuerza ejercida sobre el balón durante el lanzamiento.

Por otro lado, la composición corporal en los atletas, especialmente entre los deportistas de elite ha despertado desde antiguo gran interés en la comunidad científica. Buena prueba de ello son la gran cantidad de artículos que se han publicado describiendo el perfil antropométrico de poblaciones de diferentes deportes (Álvaro, 1993; leco, 1983; Gabbett, 2005; Bourgois, 2001; Sands, 2005; Can, 2004; Bourgois, Claessens, Janssens, Van Renterghem, Loos, y Thomis, 2001; Giordani, Cesaro, José y Silva, 2005). Las características antropométricas son parte del conjunto de variables biológicas relacionadas con el rendimiento deportivo. La cineantropometría aporta una clara información de la estructura del deportista en un determinado momento y cuantifica las modificaciones causadas por el entrenamiento. Es por ello que los factores antropométricos constituyen uno de los parámetros que orientan la identificación de talentos en diversas modalidades deportivas tanto psicomotrices como sociomotrices (Sobral, 1994; Hahn, 1988; Rodríguez, 1999; Fujii, Demura y Matsuzawa, 2005). Los estudios realizados desde los Juegos Olímpicos de 1928 han mostrado la correlación entre la modalidad deportiva que práctica el individuo y el papel de la constitución física como factor más de aptitud deportiva, existiendo en algunas modalidades un claro prototipo fisico exigido para alcanzar en un futuro a medio y largo plazo un óptimo rendimiento en el alto nivel deportivo (De Garay, Levine y Carter, 1974).

A través de los diferentes estudios, se ha tratado de definir un perfil ideal en cada deporte y gracias a ellos hoy es indudable que determinadas características físicas están ligadas al máximo rendimiento deportivo. El rendimiento óptimo requerirá por tanto, de unas ciertas características físicas que variarán en función del deporte e incluso en función de la categoría en la que se compite; de hecho parece ser que el prototipo ideal atlético propuesto hace casi un siglo, está siendo reemplazado por uno totalmente diferente, caracterizado por una alta especialización (Norton y Olds, 2001) y baraja la hipótesis de que cada deporte e incluso dentro del mismo deporte, la posición que ocupa el atleta, requiere de unos atributos físicos y fisiológicos únicos, que le permitirán obtener un alto rendimiento deportivo.

Tras revisar la literatura existente, se constata que no hay un cuerpo de conocimiento suficiente y consistente para fundamentar la evolución científica del balonmano. Las publicaciones son mínimas y las referencias bibliográficas en bases de datos científicas casi anecdóticas. Por ello se considera importante aumentar los esfuerzos de investigación, para paso a paso ir construyendo una sólida base científica.

En este contexto, este trabajo no deja de expresar las limitaciones y dificultades inherentes a esta área de investigación; no sólo en cuanto al objetivo, método y sistema de leyes científicas comunes que regulan el juego, sino también al pequeño número de trabajos que posibilitan la confrontación de motivaciones, ideas y resultados.

Este trabajo pretende cumplir principalmente dos objetivos, en primer lugar describir la estructura condicional específica de los jugadores juveniles de balonmano que militan en categoría juvenil masculina de la Región de Murcia; en segundo lugar, establecer las diferencias entre equipos.

\section{Material y método}

En este estudio participaron 45 jugadores de balonmano, que compiten en categoría juvenil en la Región de Murcia, con edades comprendidas entre los 16 y 18 años. Los clubes estudiados fueron el Alhama, el Murcia, Águilas y Jumilla Todo los deportistas y cuerpo técnico (entrenador, preparador físico,...) han sido informados de las pruebas que se van a realizar, así como de los posibles riesgos y beneficios de las mismas. Este estudio tiene carácter descriptivo y transversal.

- Se realizó una toma de datos, a mitad de temporada competitiva, para poder valorar la condición física y antropométrica en uno de los mejores momentos de forma específica, puesto que tres de los cuatro equipos se están jugando la clasificación para la copa.

Se han realizado dos valoraciones diferentes: una valoración cineantropométrica (VC) y una valoración de la condición física (VCF) .A continuación se explicarán brevemente cada una de ellas:
Tabla 1. Las variables estudiadas y los instrumentos de medida utilizados para la VC.

\begin{tabular}{|c|c|}
\hline Variables & Instrumentos de medida \\
\hline $\begin{array}{l}\text { Dimensiones corporales: peso, estatura, } \\
\text { envergadura, medida transversal de la mano } \\
\text { y perímetros del brazo contraído y medial } \\
\text { del muslo y pierna. }\end{array}$ & $\begin{array}{l}\text { Técnicas de medida recomendadas por la ISAK (Ross y } \\
\text { Marfell-Jones, 1995) y seguidas por Fragoso y Vieira } \\
\text { (2000) }\end{array}$ \\
\hline Pliegues cutáneos & Ídem anterior \\
\hline Sumatorio de tejido adiposo subcutáneo & $\begin{array}{l}\text { Sumatorio de seis pliegues }(\text { Carter, 1982 })=\text { tricipital }+ \\
\text { subescapular }+ \text { suprailíaco }+ \text { abdominal }+ \text { muslo anterior }+ \\
\text { medial pierna. }\end{array}$ \\
\hline Porcentaje graso estimado & $\begin{array}{l}\text { Yuhasz: } \\
\% \text { graso estimado }(\text { hombres })=2.585+(\Sigma 6 \mathrm{PC} \text { x } 0,1051)\end{array}$ \\
\hline Somatotipo antropométrico & (Heath y Carter, 1975) \\
\hline
\end{tabular}

Tabla 2. Variables e instrumentos de medida de la valoración condicional.

\begin{tabular}{ll}
\hline \multicolumn{1}{c}{ Variables } & \multicolumn{1}{c}{ Instrumentos de medida } \\
\hline Fuerza isométrica máxima antebrazo & Presión manual (HGR) \\
& Prueba: Dinamometría manual \\
Velocidad de lanzamiento tren superior & Lanzamiento del balón de balonmano oficial \\
& Prueba: lanzamiento con radar \\
Velocidad máxima & Carrera de velocidad \\
& Prueba: sprint $30 \mathrm{~m}$ con células fotoeléctricas \\
\hline
\end{tabular}




\subsection{La valoración cineantropométrica (VC)}

Se han analizado aquellas dimensiones corporales consideradas como importantes para el rendimiento deportivo en general y para el rendimiento en balonmano en particular (ver Tabla 1), por las diferentes fuentes bibliográficas consultadas (Cercel, 1980; Czerwinski, 1993; Álvaro, 1997; Ávila, 1996; Fernández, 1999; Laguna, 1999; Vila, 2002; Moreno, 2004).

En la realización de este estudio se han seguido las normas y técnicas de medida recomendadas por el International Working Group of Kinanthropometry (Ross y Marfell-Jones, 1991) y los criterios de la ISAK, adoptadas por el Grupo Español de Cineantropometría (GREC).

La evaluación de las características cineantropométricas se realizó estudiando aquellos parámetros que son considerados en la literatura consultada (Sobral, 1984; Malina y Bouchard, 1991; Alvaro, 1993; RFEBM, 1994; Ávila, 1996; Chirosa, 1997; Sánchez, Laguna y Torrescusa, 1997; Laguna, 1999; Fernández, 1999; Fernández, Vila, Rodríguez, Vázquez y López, 2001ª) como importantes especialmente para el balonmano (Tabla 1).

Cada uno de los miembros del equipo realizaba siempre las mismas mediciones, las cuales eran realizadas tres veces si el error no era inferior a 0.5. Con el fin de obtener el nivel de fiabilidad y precisión de las técnicas antropométricas se valoró previamente el error técnico de medida (ETM) de los medidores en un estudio con 20 sujetos. El ETM para cada una de las variables antropométricas estudiadas no superó el margen de error admitido (un cinco por ciento en los pliegues cutáneos y un dos por ciento en el resto de medidas).

\subsection{La valoración de la condición física (VCF)}

Para las pruebas de valoración funcional se utilizaron las variables más importantes relacionadas con el rendimiento (Fernández, 1999; Vila, 2002; Gorostiaga, et al, 2005) (ver tabla 2).

Para la realización de las pruebas condicionales se necesitó un material específico para cada una ellas. La disposición será en forma de circuito.

Dinamometría manual

Se valora la fuerza isométrica del antebrazo, para ello se utilizó un dinamómetro manual con empuñadura adaptable, modelo Takei Grip Dynamometer 1857.

\subsection{Velocidad máxima}

Test de velocidad con células fotoeléctricas de 30 metros, (Vicente-Rodríguez, Dorado, Pérez-Gómez, González-Henriquez, Calbet, 2004; Gorostiaga, Granados, Ibáñez, e Izquierdo, 2005). Se realiza en la pista de balonmano. Se realiza un calentamiento estandarizado que incluye cinco minutos de trote, ejercicios de estiramiento y carreras en progresión, los sujetos realizan un protocolo de series de carreras de velocidad que consiste en cuatro series de 30 metros a máxima velocidad, separados 120 segundos de recuperación. La posición inicial es la misma para todos los sujetos. El registro se realiza utilizando células fotoeléctricas elevadas del suelo 0.4 metros y colocadas a una distancia de $0.5 \mathrm{~m}, 5.5 \mathrm{~m}$ y $15.5 \mathrm{~m}$.

\subsection{Velocidad de lanzamiento de balón}

Se realizarán tres protocolos diferentes, todos desde 9 metros, el primero de los lanzamientos se realizó desde parado, otro con tres pasos previos y en apoyo y el tercero con tres pasos previos y en suspensión. Se realizaron dos intentos de cada protocolo, y se selec- cionó el mejor de los dos lanzamientos. El radar utilizado para esta valoración fué un Stalker PRO.

Para ambas valoraciones (VCy VCF) se utilizaron las instalaciones de los correspondientes a los pabellones de los diferentes equipos a valorar(Murcia, Alhama, Jumillay Águilas).

Los datos se han almacenado en una base de datos creada a tal efecto y posteriormente tratados mediante un programa estadístico comercial (SPSS para Windows, versión 12.0). Con anterioridad a dicho análisis, se realizó una depuración exhaustiva de errores de trascripción: de forma manual, en primer lugar; y mediante estadísticos y gráficos (valores medios y extremos, histogramas, diagramas de caja, etc.) en segundo lugar.

Se analizó la distribución de probabilidad de las distintas variables de estudio mediante el cálculo de estadísticos descriptivos básicos (media, desviación típica, valores extremos, etc.), y la realización de tests de hipótesis (prueba de Kolgomorov-Smirnov y Lilliefors) y prueba de homogeneidad de Levene. También se realizará un análisis de la varianza multifactorial (Anova) para establecer las diferencias estadísticas entre los distintos equipos y líneas de juego ofensivas. Se considerarán que existen diferencias significativas para $\mathrm{p} £ 0.05$.

\section{Resultados}

A continuación se presentan los resultados del estudio organizados por clubes y por líneas ofensivas.

Tabla 3. Descriptivo básico de la valoración cineantropométrica (VC) (media y desviación típica).

\begin{tabular}{|c|c|c|c|c|}
\hline Variables & Alhama (n=11) & Murcia (n=13) & Águilas (n=11) & Jumilla (n=10) \\
\hline \multicolumn{5}{|c|}{ Características generales } \\
\hline Altura $(\mathrm{cm})$ & $174.46 \pm 5.77^{1}$ & $181.10 \pm 4.89^{3}$ & $-173.33 \pm 3.73$ & $178.11 \pm 6.10$ \\
\hline Peso (kg) & $72.38 \pm 9.93$ & $86.48 \pm 14.8$ & $77.90 \pm 7.05$ & $75.06 \pm 13.16$ \\
\hline Envergadura $(\mathrm{cm})$ & $174.78 \pm 6.00$ & & $176.54 \pm 5.80$ & $181.18 \pm 6.35$ \\
\hline Mano Trns. (cm) & $23.43 \pm 1.22$ & $24.78 \pm 1.54$ & $24.32 \pm 1.58$ & $24.44 \pm 1.12$ \\
\hline \multicolumn{5}{|c|}{ Pliegues (ml) } \\
\hline Tricipital & $13.01 \pm 4.46$ & $16.21 \pm 5.61$ & $16.08 \pm 4.96$ & $13.38 \pm 4.60$ \\
\hline Subescapular & $12.88 \pm 5.33$ & $17.91 \pm 8.47$ & $14.56 \pm 4.33$ & $12.84 \pm 4.60$ \\
\hline Bicipital & $6.45 \pm 3.16$ & $8.92 \pm 4.03$ & $8.64 \pm 3.13$ & $6.96 \pm 2.45$ \\
\hline Pectoral & $7.86 \pm 2.53^{2}$ & $11.67 \pm 4.32$ & $12.42 \pm 3.89$ & $11.78 \pm 4.00$ \\
\hline Axilar & $10.37 \pm 6.15^{1}$ & $17.00 \pm 7.56$ & $14.07 \pm 4.70$ & $12.04 \pm 5.46$ \\
\hline Crestailíaca & $14.24 \pm 6.23^{1}$ & $23.25 \pm 8.04$ & $20.76 \pm 6.98$ & $16.81 \pm 7.05$ \\
\hline Supraespinal & $16.45 \pm 13.88$ & $18.88 \pm 8.46$ & $20.66 \pm 8.02$ & $16.58 \pm 9.96$ \\
\hline Abdominal & $16.80 \pm 7.05^{1,2}$ & $26.87 \pm 8.85$ & $26.69 \pm 7.71$ & $23.94 \pm 10.98$ \\
\hline Anterior Muslo & $17.63 \pm 5.30^{1}$ & $24.08 \pm 7.84$ & $21.03 \pm 6.41$ & $18.26 \pm 4.34$ \\
\hline Pierna & $11.17 \pm 3.06$ & $15.03 \pm 3.27$ & $15.05 \pm 5.47$ & $14.87 \pm 4.66$ \\
\hline \multicolumn{5}{|c|}{ Diámetros (cm) } \\
\hline Húmero & $7.08 \pm 0.43$ & $7.05 \pm 0.40$ & $7.23 \pm 0.28$ & $7.19 \pm 0.41$ \\
\hline Biestiloideo & $5.68 \pm 0.27$ & $5.64 \pm 0.25$ & $5.62 \pm 0.23$ & $5.55 \pm 0.42$ \\
\hline Fémur & $9.96 \pm 0.47$ & $10.53 \pm 0.66$ & $10.20 \pm 0.45$ & $10.16 \pm 0.35$ \\
\hline \multicolumn{5}{|c|}{ Circunferencias (cm) } \\
\hline Brazo relajado & $30.15 \pm 2.91$ & $32.77 \pm 4.53$ & $32.39 \pm 3.03$ & $28.98 \pm 3.49$ \\
\hline Brazo contraído & $31.99 \pm 1.81$ & $34.09 \pm 3.81$ & $34.10 \pm 2.08$ & $31.75 \pm 3.24$ \\
\hline Antebrazo & $26.95 \pm 1.49$ & $28.42 \pm 2.43$ & $27.88 \pm 1.38$ & $26.77 \pm 1.46$ \\
\hline Muñeca & $17.08 \pm 0.34$ & $17.48 \pm 0.70^{4}$ & $17.67 \pm 1.05$ & $16.63 \pm 0.71$ \\
\hline Tórax & $94.15 \pm 7.27$ & $100.03 \pm 9.72$ & $92.60 \pm 11.01$ & $90.28 \pm 11.72$ \\
\hline Cintura & $79.67 \pm 7.63$ & $87.17 \pm 10.30$ & $84.48 \pm 5.27$ & $80.06 \pm 7.79$ \\
\hline Glúteo & $84.59 \pm 27.85$ & $105.55 \pm 8.48$ & $102.78 \pm 6.65$ & $97.59 \pm 9.41$ \\
\hline Medial Muslo & $57.51 \pm 5.32$ & $65.65 \pm 8.54$ & $62.57 \pm 3.45$ & $59.88 \pm 7.08$ \\
\hline Pantorrilla & $53.30 \pm 4.44$ & $58.33 \pm 6.23$ & $56.30 \pm 3.78$ & $52.03 \pm 5.13$ \\
\hline Tobillo & $38.06 \pm 2.33$ & $39.36 \pm 2.90$ & $39.38 \pm 1.96$ & $37.89 \pm 2.29$ \\
\hline \multicolumn{5}{|c|}{ Porcentaje graso (\%) } \\
\hline IMC & $23.51 \pm 2.69$ & $26.28 \pm 3.90$ & $25.94 \pm 2.35$ & $23.62 \pm 3.78$ \\
\hline$\Sigma 6$ pliegues & $85.76 \pm 27.64^{1}$ & $123.86 \pm 37.05$ & $114.19 \pm 26.80$ & $100.12 \pm 34.08$ \\
\hline Yuhasz & $78.35 \pm 25.70^{1}$ & $112.50 \pm 34.40$ & $103.30 \pm 23.94$ & $89.39 \pm 30.41$ \\
\hline
\end{tabular}

Leyenda: Mano Trns. = mano transversal; ( $\left.{ }^{(}\right)$Diferencias significativas entre Alhama y Murcia; (2) Diferencias significativas entre Alhama y Águilas; $\beta$ ) Diferencias significativas entre Murcia y Águilas; $\left({ }^{4}\right)$ Diferencias significativas entre Murcia y Jumilla. 
Los resultados obtenidos muestran que el equipo de Murcia presenta una mayor estatura y un peso notablemente superior al resto de los equipos. Jumilla presenta una altura algo inferior a Murcia, mientras que Águilas y Alhama tienen una altura media similar (1,74-1,73m respectivamente). Respecto al peso, Alhama, Águilas y Jumilla obtienen medias entre 73 y 77 kilos, mientras que Murcia llega a los 86 kilos. Las medidas de envergadura igualan por una parte a Murcia y Jumilla, mientras que Alhama y Águilas están un poco por debajo $(5 \mathrm{~cm})$ de las anteriores medias.

Las medidas de la mano son similares en tres de los equipos, siendo Alhama la que obtiene unas medidas inferiores. En los valores de los pliegues se observa una igualdad entre Murcia y Águilas, siendo los resultados de Jumilla y Alhama algo inferiores.

En la tabla 3 y 4 se observa que, de todas las variables valoradas, son pocas las diferencias significativas que se aprecian entre los diferentes equipos, quizás resaltar que casi todas las diferencias se producen con el equipo de Alhama, siendo éste el único que se encuentra en la parte baja de la tabla clasificatoria.

En la figura 1 se presentan los somatotipos de los jugadores pertenecientes a los equipos estudiados. poblaciones de jugadores sesgadas por la variable altura, dentro de un mínimo elevado, que dificulta a jugadores con una altura inferior pertenecer a estos grupos.

Con respecto a la envergadura, cabe decir que su relación con la altura durante el crecimiento muestra unos valores inferiores hasta cerca de los 10 años, y a partir de aquí ambos valores tienden a permanecer parejos (Malina y Bouchard, 1991). En el presente estudio observamos que en todas las categorías la media de la envergadura resultó ser mayor que la altura, lo cual indica una longitud relativa de la envergadura mayor a la esperada dentro de los patrones normales de crecimiento. Característica importante en el rendimiento en balonmano, tanto para acciones defensivas como el uno contra uno, blocajes, etc, como para acciones ofensivas como el lanzamiento, el recibir el balón dentro de seis metros,.

Los valores de longitud transversal de la mano fueron similares en todos los equipos estudiados. Siguiendo a Malina y Bouchard (1991) sabemos que el gradiente de crecimiento en los segmentos corporales varía con respecto a la altura. En concreto, los segmentos distales (manos y pies) presentan un pico de velocidad de crecimiento anterior al de la altura y anterior al de los segmentos más proximales. Dichos autores

refieren un estudio en el que se observó que a una edad media de 16 años los jóvenes alcanzaron en la longitud de Tabla 4. Descriptivo básico de la valoración condicional (media y desviación típica).

\begin{tabular}{lcccc}
\multicolumn{1}{c}{ Variable } & $\begin{array}{c}\text { Alhama } \\
(\mathbf{n = 1 1 )}\end{array}$ & $\begin{array}{c}\text { Murcia } \\
(\mathbf{n}=\mathbf{1 3})\end{array}$ & $\begin{array}{c}\text { Águilas } \\
\mathbf{( n = 1 1 )}\end{array}$ & $\begin{array}{c}\text { Jumilla } \\
\mathbf{( n = 1 0 )}\end{array}$ \\
\hline Lanz. parado $(\mathrm{km} / \mathrm{h})$ & $69.87 \pm 5.48^{2}$ & $71.75 \pm 5.03$ & $78.45 \pm 5.80$ & $76.20 \pm 8.23$ \\
Lanz. 3 pas. $(\mathrm{km} / \mathrm{h})$ & $76.50 \pm 5.80$ & $78.87 \pm 6.55$ & $79.81 \pm 21.06$ & $80.90 \pm 6.53$ \\
Lanz. Susp $(\mathrm{km} / \mathrm{h})$. & $70.16 \pm 3.60$ & $78.33 \pm 8.73$ & & $82.60 \pm 7.44$ \\
Dinamometría $(\mathrm{N})$ & $42.55 \pm 4.56$ & $38.39 \pm 6.46$ & $40.57 \pm 5.99$ & $37.23 \pm 6.51$ \\
30 m. $(\mathrm{s})$ & $4.50 \pm 0.24$ & $4.47 \pm 0.22$ & $4.67 \pm 0.24$ & $4.53 \pm 0.21$ \\
\hline
\end{tabular}
la mano un valor próximo al $95 \%$ del tamaño final en edad adulta, mientras que en segmentos proximales como brazo y antebrazo estos valores se alcanzaron después de los 17 años (Malina y Bouchard, 1991).

Si comparamos los resultados de la longitud de la mano transversal de los jugadores de la Región de Murcia con los valores presentados por los jugadores de la selección gallega de balonmano (Fernández, 1999), encontramos que los jugadores murcianos presentan mejores valores que los gallegos (a excepción del equipo de Alhama). La longitud de la mano transversal en la categoría juvenil es ligeramente superior a los valores recomendados por la Real Federación Española de Balonmano (RFEBm) (1984) para esta categoría. Esta variable también es importante para el juego del balonmano, puesto que la adaptación y manejo del balón son determinantes para las acciones ofensivas más importantes, el lanzamiento y el pase-recepción.

En el resto de variables estudiadas, se han encontrado diferencias significativas entre el equipo de Alhamay

El componente mesomórfico es el predominante y el ectomórfico el que menores valores presentan.

\section{Discusión}

Las dimensiones corporales de altura, peso y envergadura nos dan una primera idea de las características morfológicas de los jugadores estudiados, dichos valores no presentan diferencias significativas en las variables referidas a excepción de la altura entre los equipos de Alhama y Murcia, y entre Murcia y Águilas.

Si comparamos a nuestra población con la selección gallega juvenil (Fernández, 1999), o con una selección juvenil de extremeña (De la Cruz, Sánchez, Agundez, Escalante, Feu y Saavedra, 2006) de balonmano, encontramos que tan sólo se aproximan en la variable altura el equipo de Murcia, siendo todos los demás valores inferiores a los de dichas poblaciones.

Las diferencias encontradas en la altura con preseleccionados gallegos (Fernández, 1999) y extremeños (De la Cruz, et al., 2006) de balonmano pueden ser debidas, principalmente, a que se trataban de

Tabla 5. Valores de altura, peso, envergadura y mano transversal de la selección gallega y extremeña de balonmano.

\begin{tabular}{ccccc} 
& $\begin{array}{c}\text { Altura } \\
(\mathbf{c m})\end{array}$ & $\begin{array}{c}\text { Peso } \\
\mathbf{( K g )}\end{array}$ & $\begin{array}{c}\text { Envergadura } \\
\mathbf{( c m )}\end{array}$ & $\begin{array}{c}\text { Mano Trans } \\
(\mathbf{c m})\end{array}$ \\
\hline Gallegos & 181.40 & 79.10 & 187.80 & 184.00 \\
Extremeños & 181.00 & 80.92 & - & 23.50 \\
\hline
\end{tabular}
abdominal y medial del muslo; entre Alhama y Águilas en el pliegue pectoral y abdominal. En el equipo de Murcia se han encontrado diferencias en el diámetro de la muñeca con Jumilla. De todo ello podemos interpretar que las variables antropométricas no son determinantes para los jugadores de la misma categoría, aunque estén en diferentes equipos y en lugares diferentes de la tabla.

El somatotipo que han presentado los jugadores de los cuatro equipos indican la predominancia de la mesomorfia, y valores bajos de ectomorfia, resultados que concuerdan con otros estudios (Fernández, 1999).

De las pruebas condicionales realizadas, los valores de la velocidad de lanzamiento son similares en todos los equipos, a excepción de la velocidad desde 9 metros parado en el que se han encontrado diferencias entre el equipo de Alhama y el equipo de Águilas. Las mayores velocidades se alcanzaron en los lanzamientos en los que hay desplazamiento previo (tres pasos y en apoyo y tres pasos y en suspensión), que cuando realizan lanzamientos desde parado. Hay que reseñar que en el caso de Águilas se obtienen casi los mismos valores de parado que con carrera, son el equipo que lanza a mayor velocidad.

En el apartado de valoración condicional, tan sólo se han podido comparar con otras poblaciones de jugadores de balonmano la dinamometría manual. 
Dentro de la variable fuerza isométrica máxima de tren superior, encontramos que el equipo de Alhama presenta los mejores resultados, mientras que el equipo de Jumilla los más bajos. Si se comparan estos resultados con los presentados por la selección gallega (Fernández, 1999) $(51.90 \mathrm{~N})$ y con los de la selección extremeña (De la Cruz, et al, 2006) (48.46 N), podemos comprobar que existen diferencias, siempre en detrimento de la población murciana.

En la capacidad de aceleración tampoco existen grandes diferencias entre los diferentes equipos valorados, por lo tanto a pesar de las diferencias cineantropométricas que existen entre ellos no parecen influir demasiado en los resultados de esta prueba.

\section{Conclusiones}

Las conclusiones encontradas son:

- Los equipos de la parte superior de la tabla presentan valores superiores en las variables antropométricas pero sin ser estadísticamente significativas estas diferencias.

- La envergadura es una característica importante para el rendimiento en balonmano.

- Se constata la importancia de la mesomorfia como característica predominante en el somatotipo del jugador de balonmano.

- Las mayores velocidades se alcanzaron en los lanzamientos en los que hay desplazamiento previo.

\section{Bibliografía}

Antón, J.L. (1990). Balonmano: fundamentos y etapas de aprendizaje. Madrid. Gymnos.

Álvaro, J. (1993). Perfil del jugador de balonmano. Habilidad Motriz (2), 29-32.

Álvaro, J. (1997). El acondicionamiento físico como parte del entrenamiento integrado en la etapa de la transición de Juvenil a Sénior. Problemática. Comunicación presentada en las Jornadas sobre Entrenamiento con Jóvenes en Balonmano. Alcobendas: Real Federación Española de Balonmano y Escuela Nacional de Entrenadores.

Anguera, T. (1993). Metodología observacional en la investigación psicológica. Barcelona: PPU.

Ara, I., Vicente-Rodríguez, G., Jiménez, R., Arteaga, R. y López, JA. (2003) Predicción de la altura de vuelo en el salto vertical a partir de variables antropométricas y de composición corporal en niños púberes. Selección 12 (1), $18-27$.

Aragonés, M., Casajús, J., Rodríguez, F.A. y Cabañas, M. (1993). Protocolo de medidas antropométricas. En F. Esparza, (coord.), Manual de cineantropometría. Pamplona: Federación Española de Medicina del Deporte (FEMEDE)

Ardá, A. (1998). Análisis de los patrones de juego en fútbol 7. Estudio de las acciones ofensiva. Tesis Doctoral. Universidad de La Coruña.

Ávila, F.M. (1996). Caracterización de los factores de rendimiento en balonmano. Seminario Europeo 96. Madrid: Asociación Española de Entrenadores de Balonmano.

Bayer, C. (1987). Técnica del balonmano. La formación del jugador. Barcelona: Hispano Europea.

Bosco, C. (1992). La valoración de la fuerza con el test de Bosco. Barcelona: Paidotribo.

Calbet, J.A., de Paz, J.A., Garatachea, N., Cabeza, S. y Cavaren, J. (2003) Anaerobic energy provisión does not limit Wingate exercise performance in endurance-trained cyclistis. J. Appl. Physiol. 94: 668-678.

Cardesín, J.M.; Martín, R. y Romero, J.L. (1996). Eurofit. Test europeo de aptitude fisica. A Coruña. INEF Galicia.

Carter. J.E. (1982). Body composition of Montreal Olympic Athletes. En J.E. Carter (ed.), Physical Structure of Olympic Athletes (Part I) (pp.107-116). Montreal Olympic Games Anthropological Project. San Diego: Karger.

Carter, J.E., Ross, W., Aubry, S., Hebblelinck, M. y Borms, J. (1982). Anthropometry of Olympic Athletes. En J.E. Carter (ed.), Physical structure of Olympic Athletes (Part I) (pp. 25-52). Montreal Olympic Games Anthropological Project. San Diego: Karger.

Carter, J.E.L., Ross, W., Duquet, W. y Aubry, S. (1983). Advances in somatotype methodology and analysis. Yearbook of Physical Anthropology, 26: 193-213.

Carter, J.E.L. y Heath, B. (1990). Somatotyping development and applications (pp.229-230). Cambridge: Cambridge University Press

Carvalho, C. (1996). A força em crianças e jovens. O seu desenvolvimento e treuinabilidade. Lisboa. Livros Horizonte.

Cercel, P. (1982). Balonmano. Ejercicios para las fases del juego. Bucarest: Sport -Turism.
Council of Europe. (1988). Committee for the Development of Sport: European Test of Phisical Fitness. Handbook for the Eurofit Test of Phisical Fitness. Rome: C.O.N.I.

Czerwinski, J. (1993). El balonmano: Técnica, táctica y entrenamiento. Barcelona: Paidotribo.

De Garay, A., Levine, L. y Carter, J. (1974). Genetic and Anthropological Studies of Olympic Athletes. New York: Academic Press.

Chirosa, L. J. (1997). Variables que determinan la preparación fisica en balonmano. Características, concepto y aplicación del entrenamiento complejo del juego. En I Jornadas sobre Preparación Física en los Deportes de Equipo ( $\left.{ }^{\circ} 409\right)$. Andalucía: Instituto Andaluz del Deporte.

Chirosa, R.L., Chirosa, R.I. y Padial, P.P. (2000) Efecto del entrenamiento integrado sobre la mejora de la fuerza de impulsión en un lanzamiento en suspensión en balonmano. Lecturas: Educación Física y Deportes, 5 Artículo 25. Extraído el 20 Octubre, 2005, de http://efdeportes.com.

Chirosa, L.J., Padial, P.A., Chirosa, I.J. y Doblas, J.A. (2001). El efecto de la aplicación de un método de entrenamiento de fuerza submáximo (circuittraining) sobre las diferentes manifestaciones de fuerza, a lo largo de una temporada, en un equipo femenino de balonmano. Área de balonmano 17, 1726.

Durand, M. (1992). Desenvolvemento motor e detección de xoves talentos en deporte. En el Congreso Galego do Deporte e a Educación Física Galicia: (pp. 63-69). Secretaría Xeral para o Deporte.

Espart, F. (1992). El proceso de la formación del jugador de balonmano. Etapa de iniciación global. Comunicación presentada en una conferencia impartida en Inef-Galicia: ACoruña.

Fernández, J.J. (1999). Estructura condicional en los preseleccionados gallegos de diferentes categorías de formación en balonmano. Tesis doctoral. Universidade da Coruña.

Fernández, J.J., Rodríguez, F., Vázquez, R., Vila, H. y López, P. (2001a). Multidimensional evaluation of young handball players: discrimunant analysis applied to talent selectión. En J. Mester, G. King, H. Strüder, E. Tsolakidis, A. Osterburg (Eds.), Libro de abstracts del $6^{\circ}$ Annual Congress of the European College of Sport Science \& $15^{\text {th }}$ Congress of the German Society of Sport Science, p. 1290. Cologne: ECSS, Sport und Buch Strauss.

Fernández, J.J., Vila, H., Rodríguez, F.A., Vázquez, R. y López, P. (2001b). La condición física en jugadores de balonmano gallegos en categorías de formación. II Congreso de Ciencias de la Actividad Física y el Deporte. Valencia: Facultat de Ciencies de l'Activitat Física i l'Esport.

Fleck, S.J., Smith, S.L., Craib, M.W., y Mitchell, M.L. (1992). Upper extremity isokinetic torque and throwing velocity in team handball. Appl. Sport Sci Res. 6: $120-124$

Fragoso, I. y Vieira, F. (2000). Morfología e crecimento. Lisboa: Facultade de Motricidade Humana, Universidade Técnica de Lisboa.

Fujii, K., Demura, S. y Matsuzawa, J. (2005). Optimum onset period for training based on maximum peak velocity of height by wavelet interpolation method in Japanese High School athletes. J. Physiol Anthropol Appl Human Sci 24: 1522.

García, J.A. (2003). Entrenamiento en balonmano: bases para la construcción de un proyecto de formación defensiva. Barcelona: Editorial Paidotribo.

García Manso, J. M. (1997). Hacia un nuevo enfoque teórico del entrenamiento deportivo. INFOCOES, Vol. II, (2), 3-14.

Giordani, D., Cesaro, P., José, T. y Silva, A. (2005). Morfología de atletas de handebol: comparaçao por posiçao ofensiva e defensiva de jogo. Lecturas: Educación Física y Deportes, 81 Artículo 10. Extraído el 30 Enero, 2006, de http://efdeportes.com.

Gropler, H. y Thiess, G. (1976). Der kennzeichnung der inneren struktur der koerperlichen leistungsfaehigkeiten von kinder und jugendlichen der DDR. Theorie undk Praxis der Koerperkultur, 25, (7): 543-549.

Gorostiaga, EM., Granados, C., Ibáñez, J. Gonzalez-Badillo, J.J. y Izquierdo, M. (2006). Effects of an entire season on physical fitness changes in elite male handball players. Medicine and Science in Sports and Exercise. 38(2): 357366.

Gorostiaga, E.M.; Granados, C.; Ibáñez, J. y Izquierdo, M. (2005). Differences in physical fitness and throwing velocity among elite and amateur handball players. International Journal of Sports Medicine (26): 225-232.

Gorostiaga, E.M., Izquierdo, M., Iturralde, P., Ruesta, M. y Ibáñez, J. (1999). Effects of heavy resistance training on maximal and explosive force production, endurance and serum hormones in adolescent handball players. European Journal of Applied Physiology and Occupational Physiology (80): 485-493.

González Badillo, J.J. y Gorostiaga, E. (1995). Fundamentos del entrenamiento de la fuerza. Aplicación al alto rendimiento deportivo. Barcelona: Inde.

Hahn, E. (1988). Entrenamiento con niños. Barcelona: Martínez Roca.

Heras, P. (2000). Aspectos evolutivos de la capacidad de salto: influencia de la edad cronológica de 6 a 18 años. Apunts Medicina de L'Esport. 35 (133): 19-28.

Izquierdo, M., Häkkinen, H., Gonzalez-Badillo, J.J., Ibáñez, J. y Gorostiaga, E.M. (2002). Effects of long-term training specificity on maximal strength and power of the upper and lower extremities in athletes from different sports. Eur. J. Appl. Physiol. (87): 264-271. 
Izquierdo, M., Aguado, X., Gonzalez, R., López, J.L. y Häkkinen, K. (1999) Maximal and explosive force production capacity and balance performance in men of diferent ages. Eur. J. Appl. Physiol. 79: 260-267.

Joris, H., Van Muijen, A.E., Van Ingen Schenau, G.J. y Kemper, H.C.G. (1985) Force, Velocity and energy flow during the overarm throw in female handball players. J. Biomechanics 18: 409-414.

Laguna, M. (1999). Programa de detección de talentos deportivos: concentraciones nacionales de verano 1999. Memoria. R.F.E.BM.

Lidor, R., Falk, B., Arnon, M., Cohen, Y., Segal, G. y Lander, Y. (2005). Measurement of talent in team handball: the questionable use of motor and physical tests. J. Strength Cond Res. May. 19 (2):318-25.

Loftin, M., Anderson, P., Lytton, L. Pittman, P. y Warren, B. (1996). Heart rate response during handball singles match-play and selected physical fitness components of experienced male handball players.J. Sports Med. Phys. Fitness. 36 (2): 95-99.

López, R. (1997). Los déficit de preparación. El sobreentrenamiento y su influencia en la evolución del jugador júnior. Ponencia presentada en Jornadas sobre entrenamiento con jóvenes en balonmano. La transición del jugador Juvenil a la categoría Sénior. Problemática. Alcobendas: R.F.E.BM.

Malina, R.M. y Bouchard, C. (1991). Growth, maduration and physical activity. Champaign, Illinois: Human Kinetics.

Martín Acero, R. y Vittori, C. (1997). Metodología del rendimiento deportivo (I) Sentido, definición y objeto de estudio. Revista de Entrenamiento Deportivo, Vol. XI, (1), 5-10.

Martín Acero, R. (1999). Capacidad de salto y de carrera rápida en escolares. Tesis doctoral. Tesis Doctoral. Universidade da Coruña.

Martin,A.y Ward, R. (1996). Body Composition. En D. Docherty (ed.), Measurement in Pediatric Exercise Science (pp. 87-128). Champaign, Illinois: Human Kinetics.

Mateo, J. (1990). La batería Eurofit com a mitjà de detecció de talents. Apunts Educación Física y Deportes 22: 59-68.

Mikkelsen, F. y Olsen, M.N, (1976). Handball 82-84 (Training of skudstryrken) Thesis/disertation.

Morenilla, L., López, J. y Bernetta, M. (1996). Detección y selección de talentos en gimnasia. En Consejo Superior de Deportes (Eds.), Indicadores para la detección de talentos deportivos, Vol. 3, pp. 69-104. Madrid: C.S.D.

Moreno, F. (2004). Balonmano: detección, selección y rendimiento de talentos. Madrid. Gymnos.

Neri, J. (1997). Valoración funcional de las capacidades físicas y las consecuencias para la planificación del entrenamiento. Ponencia presentada en Jornadas sobre entrenamiento con jóvenes en balonmano. La transición del jugador Juvenil a la categoría Sénior. Problemática. Alcobendas: R.F.E.BM.

Olaso, S., Martínez, J. y Planas, A. (2004). Variación de la potencia del tren inferior en jugadoras de balonmano de alta competición. Apunts. Educación Física y Deportes (76): $35-42$

Pires, C.S. (1986). Comparações antropométricas entre sexos e intraesporte na posição de jogo de jovens handebolistas brasileiros. Revista Kinesi. 2 (2): $195-$ 205

Popovic, N. y Lemaire, R. (2002). Hyperextension trauma to the elbow: radiological and ultrasonographic evaluation in handball goalkeepers. Br. J. Sports Med. (36): 452-456.

Rannou F, Prioux J, Zouhal H, Gratas-Delamarche A. y Delamarche P. (2001) Physiological profile of handball players. J Sports Med Phys Fitness. 41(3): 349-53.

Real Federación Española de Balonmano (1994). Criterios de selección de jugadoras y jugadores. Índices antropométricos, tests especificos y valores ideales. Comunicación Técnica $N^{\circ}$.1. Madrid: C.S.D. y R.F.E.BM.

Rodríguez, F.A., Gusi, N., Valenzuela, A., Nácher, S., Nogués, J. y Marina, M (1998). Valoración de la condición física saludable en adultos (I): antecedentes y protocolos de la bateríaAFISAL-INEFC. Apunts Educación Física y Deportes, (52): 54-75.

Rodríguez, F.A. (1989). Fisiología, valoración funcional y deporte de alto rendimiento. Apunts. Educación Física y Deportes (15): 48-56.

Rodríguez, F.A.(1999). Bases metodológicas de la valoración funcional. Ergometría. En. J.J. González, y J. A Villegas (coord.), Valoración del deportista: Aspectos biomédicos y funcionales. Monografias FEMEDE nº. 6, pp. 229-271. Pamplona: Federación Española de Medicina del Deporte.
Rogulj, N., Srhoj, V., Nazor, M., Srhoj L. y Cavala, M. (2005). Some anthropologic characteristics of elite female handball players at different playing positions. Coll. Antropologic. 29 (2), 705-709.

Ross, W.D. y Marfell-Jones, R. J. (1995). Cinantropometria. En J. Duncan, H MacDougall, A. Wenger y H. J. Green (eds.), Evaluación fisiológica del deportista. Barcelona: Paidotribo.

Ruiz, L. M. (1994). Deporte y aprendizaje. Procesos de adquisición y desarrollo de habilidades. Madrid: Visor Distribuciones.

Saavedra, J. M. (2002). Valoración multidimensional y rendimiento en nadadores jóvenes de nivel nacional. Tesis Doctoral. Universidade da Coruña.

Saavedra, J.M., Escalante, Y. y Rodríguez, F.A. (2003). Multidimensional evaluation of peripuberal swimmers: multiple regression analysis applied to talent selection. En J.C. Chatard (ed.), Biomechanics and Medicine in Swimming, $I X$, pp. 551556. Saint-Étienne: Publications de l'Université de Saint-Étienne.

Sánchez Sánchez, F., Laguna, M. y Torrescusa, L. C. (1997). Programa de detección, perfeccionamiento y seguimiento de talentos deportivos. Concentraciones infantiles: Memoria. Madrid: Real Federación Española de Balonmano y Consejo Superior de Deportes.

Seirul-lo, F. (1990). Entrenamiento de la fuerza en balonmano. Revista de Entrenamiento Deportivo 4 (6), 30-34.

Seirul-lo, F. (1993). Preparación fisica aplicada a los deportes de equipo: Balonmano. Cuaderno Técnico Pedagógico nº 7. A Coruña: Centro Galego de Documentación e Edicións Deportivas.

Silla, D. y Rodríguez, F. (2005). Valoración de la condición física en jugadores de hockey hierba de alto nivel. Apunts. Educción Física y Deportes (80): 37-44.

Sobral, F. (1986). Curso de antropometría. Lisboa: ISEF

Sobral, F. (1994). O Andebol à Medida da Crianca. Andebol Revista (1): 8-11.

Solanellas, F. (1995). Valoración funcional de tensitas de diferentes categorías. Tesis doctoral. Universidad de Barcelona.

Solanellas, F. y Rodríguez, F.A. (1996). Valoración de la condición física de tensitas de diferentes categorías. $8^{\text {th }}$ FIMS European Sports Medicine Congress, p. 126. Granada: Universidad de Granada.

Srhoj V, Rogulj N, Zagorac N, Katic R. (2006). A new model of selection in women's handball. Coll Antropol. 30 (3): 601-5.

Srhoj, V. (2002). Situational efficacy of anthropomotor types of young female handball players. Coll Antropol. 26 (1): 211-218.

Srhoj, V., Marinovic, M. y Rogulj, N. (2002). Position specific morphological characteristics of top-level male handball players. Coll Antropol. 26 (1): 219227

Toumi H, Best TM, Martin A, y Poumarat G. (2004). Muscle plasticity after weight and combined (weight + jump) training. Med Sci Sports Exerc. 36 (9): 15808.

Tyrdal, S. y Bahr, R. (1996). High prevalence of elbow problems among goalkeepers in European team handball - «handball goalie's elbow». Scand. J. Med. Sci. Sports (6): 297-302.

Van Muijen, A.E., Joris, H., Van Ingen Schenau, G.J. y Kemper, G.J. (1991). Throwing practice with different ball weights: effects on throwing velocity and muscle strength in female players. Sports Training, Med. y Rehab. 2: 103-113.

Verjoshanski, I. (1990). Entrenamiento deportivo: planificación y programación. Barcelona: Martínez Roca.

Vicente-Rodríguez, G., Dorado, C., Pérez-Gómez, J., González-Henriquez, J.J. y Calbet, J.A. (2004). Enhanced bone mass physical fitness in young female handball players. Bone 35: 1208-1215.

Vieira F. y Fragoso, I. (1999). Perfil morfológico de atletas femeninas portuguesas de diferentes modalidades. Universidade Técnica de Lisboa: Facultade de Motricidade Humana

Vila, Ma. H. (2002). Estructura condicional en las preseleccionadas gallegas de diferentes categorías de formación en balonmano. Tesis doctoral. Universidade da Coruña.

VV.AA. (1998). Balonmano: el lanzamiento en suspensión. A Coruña: INEF Galicia.

Wallace, M.B. y Cardinale, M. (1997). Conditioning for team handball. Strength y Conditioning. December: 7-12.

Zatsiorski, V.M. (Ed) (1989). Metrología deportiva. Moscú y La Habana: Planeta y Pueblo.

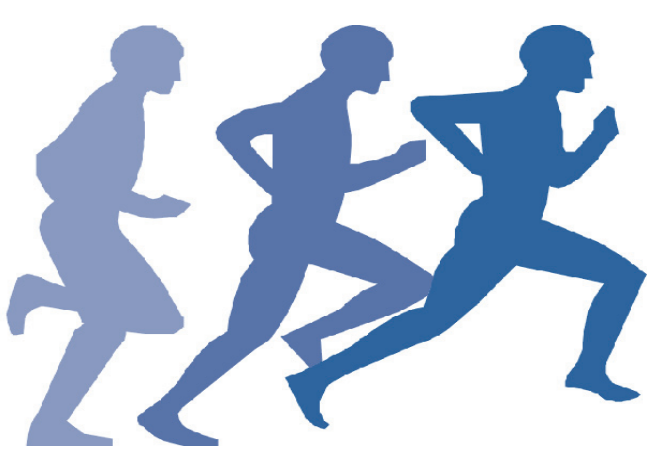

